\title{
New Perspective in Osteoarthritis: The OPG and RANKL System as a Potential Therapeutic Target?
}

\author{
Steeve Kwan Tat, ${ }^{1}$ Jean-Pierre Pelletier, ${ }^{1}$ Carmen Ruiz Velasco, ${ }^{2}$ \\ Marc Padrines $^{2}$ and Johanne Martel-Pelletier ${ }^{1}$ \\ ${ }^{1}$ Osteoarthritis Research Unit, University of Montreal Hospital Centre, \\ Notre-Dame Hospital, Montreal, Quebec, Canada \\ ${ }^{2}$ Université de Nantes, Laboratoire de Physiopathologie de la Résorption Osseuse et Thérapie des \\ Tumeurs Osseuses Primitives, Nantes Cedex 01, France
}

(Received for publication on June 24, 2008)

(Revised for publication on September 3, 2008)

(Accepted for publication on October 9, 2008)

\begin{abstract}
Bone remodelling is tightly regulated by a molecular triad composed of OPG/RANK/RANKL. The receptor activator of NF- $\mathrm{KB}$ ligand (RANKL) (localized on osteoblasts) enhances osteoclastogenesis via interaction with its receptor RANK (localized on osteoclasts), whereas osteoprotegerin (OPG) (produced by osteoblasts) inhibits this osteoclastogenesis by binding to RANKL. The equilibrium between OPG and RANKL plays a crucial role in the pathophysiology of bone. Although some studies have shown the efficacy of OPG as a therapeutic agent against bone resorption, its bioavailability and mechanism of action after binding to RANKL have only recently been studied. A mechanistic investigation based on what becomes of OPG after binding to cells expressing membranous RANKL demonstrated an internalization process of OPG through the clathrin pathway prior to proteasomal and/or lysosomal degradation. Interestingly, the OPG internalization process reduced the half-life of RANKL. Recent evidence has shown that subchondral bone alterations in osteoarthritis (OA) are intimately involved in cartilage degradation, and that OPG and RANKL may be implicated. Data show that human OA subchondral bone osteoblasts have abnormal OPG and RANKL levels and consequently an altered OPG/RANKL ratio. Further data also reveal the involvement of some osteotropic factors in these altered levels and that some of these factors generally target RANKL with a differential modulation of the RANKL isoforms. Altogether, data suggest that this system could be targeted as a new strategy for the treatment of OA. (Keio J Med 58 (1) : 29-40, March 2009)
\end{abstract}

Keywords: OPG, RANKL, osteoarthritis, osteoblasts, subchondral bone

\section{Introduction}

Bone is a specialized connective tissue made up of several components including the specific cells osteoblasts, osteocytes and osteoclasts, inorganic non-collagenic substances such as proteoglycans, and a collagenic component of which collagen type I is the major constituent. Bone remodelling involving bone matrix formation and degradation occurs through osteoblast and osteoclast activity The equilibrium between the activities of these two cells maintains the mineral homeostasis via the release of calcium and phosphorous molecules into the circulation (Fig. 1). ${ }^{1}$ The bone remodelling cycle includes several distinct phases, the main ones being activation, resorption, reversal and formation. Physiological events such as mechanical force and stress induce bone remodelling, in which osteoclast precursors and mature osteoclasts are recruited from the circulation to the bone re-

Presented at the 1559th meeting of the keio Medical Society in Tokyo, April 25, 2008

Reprint request to: Johanne Martel-Pelletier, PhD, Osteoarthritis Research Unit, University of Montreal Hospital Centre, Notre-Dame Hospital, 1560 Sherbrooke Street East, Montreal, Quebec, Canada, H2L 4M1, Tel: +1-514 8908000 (ext. 26658), Fax: +1-514 412 7582, Email: jm@martelpelletier.ca 

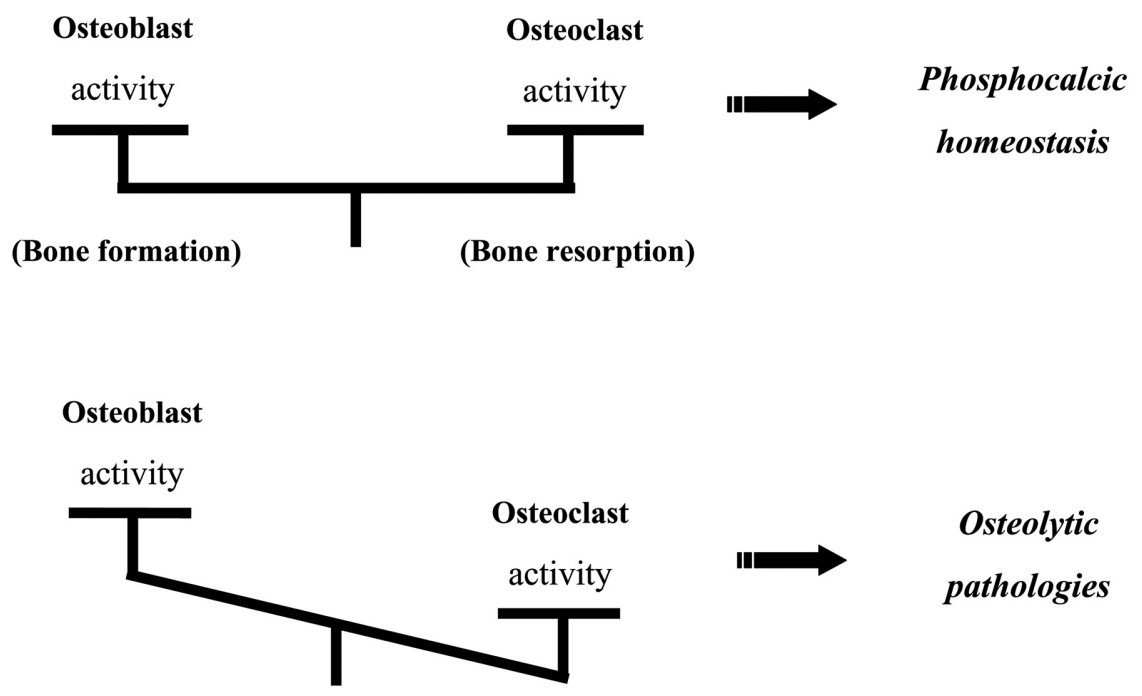

Fig. 1 Bone remodelling is associated with osteoblast activity in bone formation and osteoclast activity in bone resorption. Such equilibrium maintains the phosphocalcic homeostasis. Imbalance favouring excess osteoclast activity is often described during various osteolytic pathologies.

modelling unit, thereby initiating the resorption of the mineralized matrix. Following this process, the osteoclasts undergo apoptosis, leaving an opening for osteoblasts to deposit newly synthesized matrix. ${ }^{2}$

Bone remodelling takes place through a coordinated action between osteoclasts and osteoblasts. Osteoclasts are multinucleated giant cells deriving from the hematopoietic progenitors of the monocyte-macrophage lineage. ${ }^{3}$ Their main function is to resorb the mineralized tissues of the bone microstructure. Osteoclasts can be distinguished by their specific morphology and features such as a polarized morphology and an apical differentiation adjoining the calcified matrix in resorption (ruffled border). These cells are responsible for the acidification of the extracellular matrix before the release of proteolytic enzymes involved in the degradation of bone tissues. ${ }^{3}$ Osteoblasts are derived from the bone marrow cells and are responsible for the synthesis of new matrix. These cells are progressively transformed into osteocytes when trapped in their own secreted matrix. ${ }^{4}$ Secondary cell types such as monocytes/macrophages and endothelial cells also contribute to bone remodelling by acting either directly with the osteogenic cells or indirectly through the release of soluble factors, including cytokines, growth factors, and others.

The bone remodelling process becomes unbalanced in a variety of pathologic situations, thereby affecting the general skeleton structure. Diseases such as post-menopausal osteoporosis, bone metastasis, and rheumatoid arthritis include local and/or systemic alterations in which the level of hormones and pro-inflammatory cytokines known to stimulate or inhibit bone resorption are highly involved (Fig. 1). ${ }^{5}$ In this context, bone stimuli (mechan- ical load, hormones, and growth factors) can act directly on osteogenic cells as well as on their precursors to control the differentiation, formation and functions (matrix formation, mineralization, resorption, etc.) of these cells.

This review focuses on OPG (osteoprotegerin), RANK (receptor activator of nuclear factor- $\kappa \mathrm{B}$ ) and RANKL (RANK ligand), a molecular triad that has been demonstrated to be of particular importance in the control of bone pathophysiology. The molecular and cellular mechanisms of action of OPG and RANKL will be reviewed. Moreover, this triad will be addressed in the context of osteoarthritis (OA). As substantial evidence is now available suggesting that subchondral bone remodelling prevails early in the disease process, the involvement of OPG and RANKL in the subchondral bone during OA will be reviewed. Finally, the possible involvement of OPG and RANKL as specific therapeutics for OA will also be discussed.

\section{OPG/RANK/RANKL Molecular Triad}

It has been shown that some members of the tumor necrosis factor (TNF) superfamily play major roles in regulating bone metabolism. The molecular triad composed of OPG/RANK/RANKL, members of this superfamily, has been described as a key cytokine system for controlling the differentiation and function of osteoclast biology. ${ }^{5}$ Moreover, independent of the bone system, studies have also revealed new and important functions of this triad in other pathologies and tissues including mammary gland development and lactation, the immune and vascular systems, and the proliferation of cancer cells. ${ }^{5}$ 


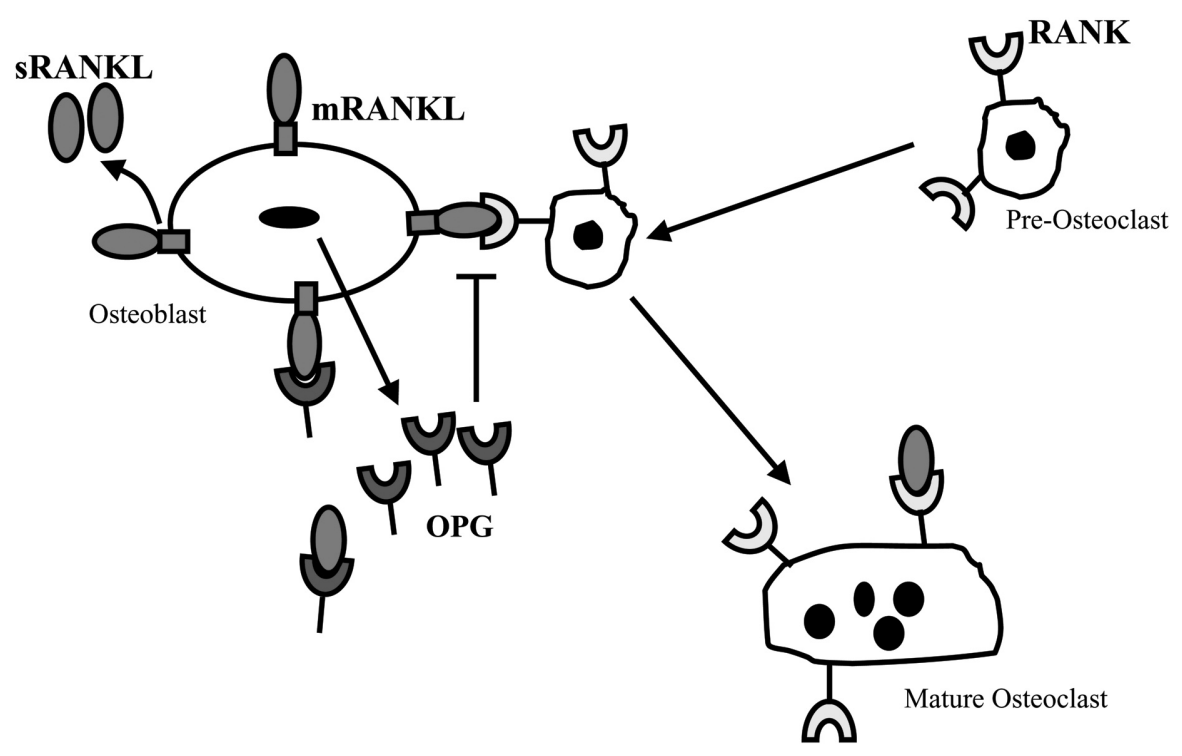

Fig. 2 OPG/RANK/RANKL molecular and cellular mechanisms of action. RANKL is produced by the osteoblast cells in membranous (mRANKL) or soluble (sRANKL) form. Its receptor RANK is expressed on the cell surface of pre-osteoclastic cells and the interaction between RANK-RANKL induces the differentiation and formation of multinucleated mature osteoclasts. The third protagonist, OPG is also produced by the osteoblasts and exerts an inhibitory effect on the pre-osteoclast differentiation process. OPG, by binding to RANKL, inhibits the interaction of RANK-RANKL and the subsequent osteoclastogenesis. Hence, RANKL is described as a pro-resorptive and OPG as an anti-resorptive agent.

\section{OPG/RANK/RANKL Cellular and Molecular Mechanisms}

In the bone remodelling process, the members of the molecular triad OPG/RANK/RANKL are closely linked to each other. RANKL is synthesized either in membranous or soluble form, primarily by the osteoblastic lineage cells, the immune cells and some cancer cells. This factor is essential for mediating bone resorption through osteoclastogenesis and the activation of mature osteoclasts. RANKL stimulates osteoclastogenesis and osteoclast activity by binding to the cell surface receptor RANK, located on osteoclasts (precursor and mature). ${ }^{6,7}$ The binding of RANKL to the extracellular RANK leads to the activation of specific signalling pathways involved in the formation and survival of osteoclasts, hence bone resorption. ${ }^{8,9}$ OPG is secreted by the stromal cells and other cell types, including osteoblasts, and acts as a soluble decoy receptor for RANKL. OPG, by interacting with RANKL, inhibits the binding of RANKL to RANK, thereby preventing RANK activation and subsequent osteoclastogenesis and, as a result, inhibits bone resorption (Fig. 2). ${ }^{10}$

In the immune system, the molecular and cellular mechanisms of this triad are important for maintaining an immune response. In this system, RANKL expressed by activated T cells interacts with RANK, which is expressed by the dendritic cells, and such binding of RANKL to RANK on dendritic cells regulates the function and survival of these cells. ${ }^{11-13} \mathrm{OPG}$, on the other hand, is produced by the B lymphocytes as well as dendritic cells and maintains an equilibrium in this system. The OPG/RANK/RANKL system, however, is not the only cytokine mechanism that controls the immune response. Indeed, the cross-talk between $T$ cells and dendritic cells also involves CD40 and CD40L interaction. $^{12,13}$

There is accumulating evidence of the potential role of OPG and RANKL in other tissues including the vascular system. OPG and RANKL have been found to be associated with pathologies such as coronary artery disease, atherosclerosis and vascular calcification in patients. Indeed, experiments done on OPG knockout mice have revealed the anti calcification property of OPG. ${ }^{14-16}$ In this system, the absence of OPG favours the medial calcification of the aorta as well as calcification of the renal arteries. Treatment of these knockout mice with OPG significantly reduced the calcified lesion areas.

\section{$O P G$}

OPG belongs to the TNF receptor (TNFR) superfamily, but differs from the common membranous TNFR in that it is a soluble receptor. ${ }^{17}$ OPG functions as an endogenous antagonist receptor that prevents the biological effects of RANKL, both membranous and soluble, and thus acts as an inhibitor of bone remodelling/resorption. OPG is highly expressed in the adult lung, heart, kidney, liver, thymus, lymph nodes and bone marrow, and is syn- 
thesized by several cells including stromal cells, osteoblasts, vascular smooth muscle cells, B lymphocytes, and articular chondrocytes. ${ }^{18,19}$ OPG is produced as a proprotein of 401 amino acids and undergoes an intracellular cleavage resulting in a final 380 amino acids mature protein. ${ }^{6}$

OPG production by specific cells is modulated by several cytokines, vitamins, estrogens and other molecules, thereby modulating osteoclastogenesis and bone resorption. OPG production is induced by $1 \alpha, 25$-dihydroxyvitamin $\mathrm{D}_{3}$, estrogens, pro-inflammatory cytokines such as interleukin-1 (IL-1) and TNF- $\alpha$ as well as transforming growth factor- $\beta$ (TGF- $\beta$ ), whereas parathyroid hormone (PTH) and glucocorticoids inhibit OPG production. ${ }^{20-26}$ The importance of OPG was illustrated on OPG-deficient mice, in which osteoclast formation was greatly enhanced, and also on an overexpressing-OPG mouse model, in which severe osteopetrosis with reduced numbers of osteoclasts was observed. ${ }^{18,27}$

Moreover, OPG can directly inhibit osteoclast activity, independently of RANKL, through interactions with still uncharacterized receptors present on osteoclasts. ${ }^{28}$ The biological effects of OPG on bone cells include the inhibition of the terminal stages of osteoclast differentiation, suppression of mature osteoclast activation and the induction of apoptosis of these cells.

\section{RANK}

RANK, like OPG, belongs to the TNFR superfamily. It is synthesized as a type I transmembrane protein of 616 amino acids and assembles itself into a functional trimer. ${ }^{29}$ RANK is considered a natural receptor of RANKL and is also expressed by several different tissues such as skeletal muscle, thymus, liver, colon, mammary glands, prostate, pancreas, and cells of the monocyte/macrophage lineage including precursors and mature osteoclasts, B and T lymphocytes, dendritic cells, fibroblasts, and articular chondrocytes. ${ }^{6}$ Activation of RANK, like other TNFR-related proteins, induces a cascade of intracellular signalling factors including the recruitment of TNFR-associated factor proteins (TRAF), the transcription factors NF- $\kappa B$ subunits $\mathrm{p} 50$ and $\mathrm{p} 52$, AP-1 and nuclear factor of activated T cells (NFAT), the mitogen-activated protein (MAP) kinase cascades including ERK1/2, JNK and p38, and the induction of Srcand phosphatidylinositol 3- kinase-dependent Akt activation. ${ }^{30}$

The cytoplasmic domain of RANK possesses independent TRAF interaction motifs that bind to TRAF-2, -5 and -6 . RANK mutants and selective deletions of RANK demonstrated that TRAF-6/RANK interaction is the main adaptor molecule for RANK signalling. ${ }^{9}$ With TRAF-2 and TRAF-5 deficiency, only a slight reduction in osteoclastogenesis was observed, thus showing their minor participation in controlling osteoclast formation. ${ }^{31,32}$ Similar to TRAF-6 deficiency, mice expressing neither RANK nor the NF- $\kappa B$ subunits $\mathrm{p} 50$ or p52, developed severe osteopetrosis due to a total absence of osteoclasts. ${ }^{33,34}$ NFAT, identified as one of the downstream targets of RANK activation, is increased in the presence of RANKL, and in cooperation with c-Fos leads to the terminal differentiation of the osteoclasts by promoting the expression of osteoclast-specific genes such as tartrate-resistant acid phosphatase (TRAP) and calcitonin receptors, and its lack leads to an inhibition of osteoclast numbers. $^{35,36}$

Hence, the activation of RANK stimulates the differentiation of osteoclastic precursors into mature osteoclasts as well as the activation of mature osteoclasts. RANK activation by RANKL involves distinct signalling cascades and mice ablated of these components were found to have an osteopetrotic phenotype.

\section{$R A N K L$}

RANKL belongs to the TNF superfamily and is synthesized as a mature protein of 317 amino acids. RANKL is largely expressed in bone, bone marrow and lymphoid tissues. $^{37-39}$ The predominant roles of RANKL in bone physiology are the stimulation of osteoclast differentiation/activation and the inhibition of osteoclast apoptosis. ${ }^{6}$ In addition, this cytokine in association with macrophage-colony stimulating factor (M-CSF) is involved in the osteoclastogenesis process. The importance of RANKL was demonstrated in RANKL knockout mice which presented severe osteopetrosis and total loss of osteoclasts. $^{27}$

Human RANKL has recently been shown to exist as 3 isoforms: RANKL1, 2, and 3. RANKL1 and 2 encode for transmembrane forms with an absence of intracellular domain for human RANKL2, whereas RANKL3 lacks the intracellular and transmembrane domain. ${ }^{40}$ Murine RANKL has also been described to exist in 3 isoforms; however, murine RANKL2 possesses a short cytoplasmic domain, which is completely absent in human RANKL2. These different isoforms are able to differentially regulate osteoclastogenesis. ${ }^{40-42}$ In cells, RANKL forms homo- or heterotrimer structures between the isoforms, ${ }^{43,44}$ and the trimeric combination of the isoforms is crucial for orientating the RANKL membranous localization, thereby controlling the osteoclast formation and differentiation process. Hence, the trimeric structure of RANKL1 alone or in association with RANKL2 is translated into a membranous localization. When RANKL1 or 2 is co-transfected with RANKL3, however, a reduced level of membranous localization is observed, indicating that RANKL3, by preventing the membranous localization of RANKL, acts as an inhibitor of osteoclastogenesis. ${ }^{40-42}$ Moreover, NIH3T3 cells 


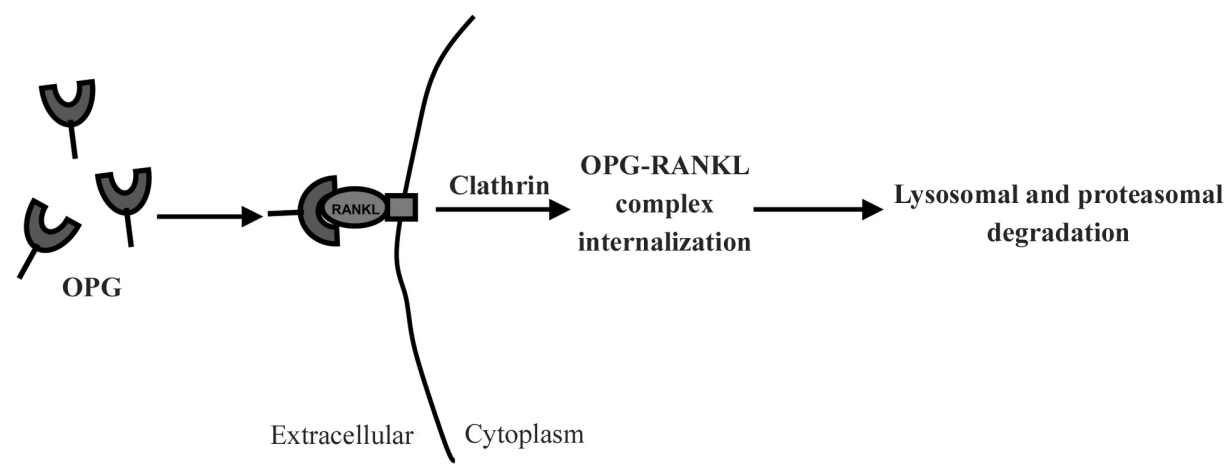

Fig. 3 Exogenous OPG is internalized through the clathrin pathway by membranous RANKL before being degraded via the proteasome and the lysosome.

transfected with human RANKL2 alone were unable to induce osteoclastogenesis, whereas those transfected with murine RANKL2 induced osteoclastogenesis. Such difference could be explained by the fact that murine RANKL2 possesses an intra-cytoplasmic domain allowing the membranous localization of RANKL whereas an absence of such intra-cellular domain for trimerized human RANKL2 may explain its inability to be expressed at the cell surface. ${ }^{40,42}$

RANKL exists in both a soluble and a membranous form. ${ }^{41}$ The soluble form of RANKL, which corresponds to the C-terminal domain of membranous RANKL, may be produced either directly by the cell through an alternative splicing followed by a secretion extracellularly or by a proteolytic cleavage of membranous RANKL. So far, various proteases have been proposed to be involved in the shedding process. Among these, the tumor necrosis factor converting enzyme (TACE), the membranous metalloprotease MT1-MMP, MMP-7, and ADAM-19 have all been shown to proteolytically cleave membranous RANKL. ${ }^{45-49}$

\section{THE OPG-RANKL Molecular Complex}

Bone remodelling is tightly controlled by OPG and RANKL; however, the ratio of OPG/RANKL is considered to better reflect environmental signals. A high ratio of OPG/RANKL is indicative of promoting bone formation while a low ratio favours bone resorption. Such imbalance has been encountered in some osteolytic lesions where RANKL is up-regulated as well as in osteoblastic disorders where OPG is up-regulated. ${ }^{50}$

Although several studies have proven the beneficial effect of administering OPG as a specific therapeutic agent against bone resorption as well as against arterial calcification in cardiovascular disorders, there is very little data concerning the intimate modulation of exogenous OPG bioavailability in the extracellular environment. It has been shown that OPG, through its heparin binding domain interacts with some proteoglycans such as syndecan-1 and that OPG binding to cells expressing high levels of membranous syndecan-1 is internalized, then degraded. ${ }^{51,52}$ Similarly, with RANKL expressing cells, the binding of OPG to membranous RANKL is also internalized, yet through another process, the clathrin coat formation pathway, which occurs before proteasomal and lysosomal degradation (Fig. 3). ${ }^{53}$ Thus, these two OPG internalization mechanisms, through the lipid rafts by membranous syndecan- 1 or the clathrin pathway, by membranous RANKL are involved in the control of the bioavailability of extracellular OPG. Interestingly, on RANKL expressing cells, OPG was also demonstrated to regulate the half-life of membranous RANKL; by binding to RANKL, OPG induces the degradation of RANKL. ${ }^{53}$

Further investigations on the effect of exogenous soluble glycoaminoglycans (GAGs) on the binding of OPG to RANKL showed that GAGs such as heparin, heparan sulfate, chondroitin sulfate and dermatan sulfate bind to OPG via the heparin binding domain. Such OPG-GAG binding inhibits OPG-RANKL interaction, thereby preventing OPG internalization mediated by membranous RANKL. In vivo, GAGs and proteoglycans of the microenvironment could interact with endogenous OPG, thereby modulating the bioavailability of OPG and RANKL. ${ }^{54}$

In most of the cytokine-cytokine receptor systems, such as the TNF/TNFR family, the cytokine interacts with its transmembranous receptor before being internalized. Thus, OPG-RANKL interaction represents an original mechanism in which OPG, being the receptor, is internalized by its ligand RANKL. This internalization process is of particular importance for future therapeutic involvement of OPG. In fact, although OPG has been successfully used as a therapeutic anti-resorptive agent against osteolytic diseases such as postmenopausal osteoporosis, multiple myeloma, tumor associated humoral 
hypercalcemia and rheumatoid arthritis, ${ }^{55-58}$ the decrease in extracellular OPG concentration observed in RANKL-expressing cells raises the question of its bioavailability during osteolytic disease therapies, especially when RANKL is abundantly expressed. In this particular situation, denosumab, a fully human monoclonal antibody against RANKL, is a potent anti-resorptive agent. ${ }^{59}$ Its inhibition of the RANK-RANKL interaction as well as the OPG internalization, thereby enhancing the circulating concentration and bioavailability of endogenous OPG, could contribute to an additive effect in inhibiting bone resorption.

The OPG-RANKL mechanism is much more complex than previously thought. Indeed, the anti-resorptive effect of OPG can be explained not only by its properties as a decoy receptor (competitor inhibitor of RANK/ RANKL binding) but also as a modulator of RANKL half-life and the RANKL shedding mechanism process. As RANKL controls the bioavailability of OPG and vice versa, the equilibrium between membranous RANKL and soluble OPG will be determinant of a curative application of OPG. Approaches should target the maintaining of an optimal soluble extracellular OPG concentration by reducing OPG internalization.

\section{Osteoarthritis}

The articular joint is made up of several tissues, the main ones involved during osteoarthritis (OA) being the cartilage, synovial membrane, and the subchondral bone, all of which are closely linked. The cartilage is the tough elastic material that covers and protects the ends of bone. In a healthy joint it acts as a shock absorber when weight is exerted on the joint and its slippery surface allows the joints to move smoothly. Joint degeneration does occur, however, and OA is among the most common joint disorders, affecting about $65 \%$ of individuals over 60 years of age. The disease causes pain and functional disability, resulting in a significant social and economic burden. Despite the high prevalence of this disease, its precise etiopathogenesis is not yet completely understood, although significant progress has been made during the last few decades. The joints commonly affected by OA are the base of the thumb, the end joints or middle joints of the fingers, the hips, the knees and the joints at the base of the big toe, although the neck (cervical spine) and the lower back (lumbar spine) can also be affected.

$\mathrm{OA}$ is considered a complex illness in which all the tissues of the joint play significant roles. ${ }^{60}$ Even though articular cartilage degeneration is a major characteristic of OA, we still do not know what initiates it. Synovial membrane inflammation is believed to play an important role in the progression of joint tissue lesions; however, there is a general consensus that synovial inflammation in $\mathrm{OA}$ is not the primary cause of the disease, but rather a secondary phenomenon related to multiple factors including cartilage matrix degradation. Moreover, studies have confirmed that the subchondral bone during OA is the site of several dynamic morphological changes that appear to be part of the disease process. ${ }^{61}$ These changes are associated with a number of local abnormal biochemical pathways related to the altered osteoblast metabolism in this tissue.

Some clinical studies in OA patients have suggested that the indices of bone resorption are increased early in the disease process. This concurs with the in vivo findings of a study performed on an induced OA dog mod$\mathrm{el}^{62}$ allowing the chronological analysis of the disease progression. At an early stage of the disease process, increased bone loss and resorption with subchondral bone exhibiting surface and trabecular thickness reduction and an increased number of osteoclasts, as well as production of catabolic factors including cathepsin K and MMP-13 were observed. ${ }^{62}$ Moreover, an in vivo experiment performed at an early stage on the same dog model demonstrated an inhibition of the subchondral bone loss upon treatment with bisphosphonates (an inhibitor of osteoclast function). ${ }^{63}$ Also reported on this dog model at a later stage, 54 months after OA induction, was that the histomorphometric analysis of the subchondral bone revealed a marked increase in volume with active bone formation. ${ }^{64}$

Human OA subchondral bone osteoblasts have demonstrated abnormal phenotypes, including elevated alkaline phosphatase activity and increased release of osteocalcin. ${ }^{65}$ Other factors such as TGF- $\beta$, insulin-like growth factor-1 (IGF-1) and the urokinase-type plasminogen activator ( $\mathrm{UPA}$ )/plasmin system have also been found at abnormal levels in OA subchondral bone. ${ }^{66}$ Moreover, our group has also reported that OA subchondral bone osteoblasts, which otherwise demonstrate no different phenotypic features, can be discriminated into two subgroups identified by their level (low [L] or high $[\mathrm{H}]$ ) of endogenous prostaglandin $\mathrm{E}_{2}\left(\mathrm{PGE}_{2}\right) \cdot{ }^{67-71}$ Hence, $\mathrm{OA}$ patients can be discriminated into groups named L-OA or H-OA.

\section{Involvement of OPG and RANKL during Osteoarthritis}

In OA, a subchondral bone resorption/formation process has been shown to occur, in which there are phases of bone degradation and others of bone formation. Interestingly, factors such as OPG and RANKL, which constitute specific components capable of influencing the bone remodelling process, have been found to be expressed and modulated in human OA subchondral bone. In addition to subchondral bone, the OPG/RANK/ RANKL triad has also been observed to be expressed by the other articular cells. Indeed, articular chondrocytes 
also express each factor of the triad but with specific localization in the cartilage, which depends on whether the cartilage is normal or diseased. OPG, RANK and RANKL have been detected in the superficial zone of normal cartilage whereas during OA their expression was found to extend to the middle zone. ${ }^{72}$ At present, however, the functional consequence of their expression by the articular chondrocytes remains unknown. Nevertheless, besides the mechanism of action of OPG binding to RANKL thus decreasing the RANKL activity, a recent study on an OPG transgenic mouse model of OA suggested that the in vivo beneficial effect of the administration of OPG could also be due to its capacity to bind tumor necrosis factor-related apoptosis-inducing ligand (TRAIL), an inducer of chondrocyte apoptosis. Hence, OPG, by binding to TRAIL, is able to prevent chondrocyte apoptosis. ${ }^{73}$ Of note, the beneficial anti-apoptotic effect of OPG on some cells could be somewhat detrimental when applied to other systems. For example, OA as well as rheumatoid arthritic synovial membrane demonstrate hyperplasia, and one of the envisaged therapeutic strategies involved increasing apoptosis of cells in this tissue, in which case, the anti-apoptotic effect of OPG would be unbeneficial as it could further increase the number of cells in the synovial membrane.

Synovial cells have also been observed to express OPG and RANKL, and RANKL appears actively involved in the erosion of bone during rheumatoid arthritis. ${ }^{74-76} \mathrm{In}$ the presence of cytokines commonly involved during rheumatoid arthritis, synoviocytes were able to induce osteoclastogenesis by up-regulating the production of RANKL. ${ }^{74,75,77}$ Moreover, synovial fluid of rheumatoid arthritis patients showed an induced osteoclast formation due to excessive production of RANKL. ${ }^{78}$ Such regulation of the OPG/RANKL ratio favours destruction of mineralized tissue including the cartilage and the cortical bone. In vivo studies performed on animal models of arthritis have proven the beneficial effect of OPG injection at reducing cortical bone erosion. ${ }^{79,80}$ In another arthritic disease, psoriatic arthritis, dysregulation of OPG and RANKL toward an elevation of RANKL was also shown. ${ }^{81,82}$

Although these factors are gaining importance in the progression of inflammatory joint diseases (rheumatoid arthritis and psoriatic arthritis), their participation in OA has until now been unclear. Recently, efforts have been devoted to a better comprehension of the implication of OPG and RANKL during the different stages of OA. Improved understanding is without doubt of major importance in better identifying a specific strategic treatment for this disease. It was recently demonstrated that during longstanding OA and rheumatoid arthritis, the OPG/ RANKL ratio in the synovial fluid is much more elevated in OA compared to rheumatoid arthritis. ${ }^{83,84}$ Hence, cortical bone destruction is much more prevalent in rheu- matoid arthritis compared to OA, an observation which is correlated with clinical observation. In this latter study, however, the remodelling process of the subchondral bone was not evaluated. This data could have been of great importance in establishing a close relationship between the OPG/RANKL ratio and the subchondral bone changes.

As described previously, studies performed on OA patients revealed two distinct phenotypes of subchondral bone osteoblasts, L-OA and H-OA. ${ }^{67-71}$ In situ histological examination of the subchondral bone revealed that the thickness of this tissue was reduced in the L-OA and increased in the H-OA patients compared to normal individuals. ${ }^{70}$ Further experiments in which the bone remodelling factors OPG and RANKL were determined in OA subchondral bone osteoblasts compared to normal revealed that in both the L-OA (having low endogenous levels of $\mathrm{PGE}_{2}$ ) and $\mathrm{H}-\mathrm{OA}$ (having high endogenous levels of $\mathrm{PGE}_{2}$ ) osteoblasts, OPG and RANKL were abnormally expressed and consequently the OPG/RANKL ratio was reduced in the L-OA, suggesting increased subchondral bone resorption, and increased in H-OA, indicating a shift toward bone formation. ${ }^{70}$ These data were further strengthened by an osteoclastogenesis experiment in which the L-OA osteoblasts induced in the pre-osteoclastic murine cell line RAW 264.7 a significantly higher level of mature osteoclasts compared to the H-OA. ${ }^{70}$ These findings are summarized in Figure 4.

Further studies carried out on the L-OA and H-OA osteoblasts investigated the membranous localization of RANKL, and the expression level of RANKL isoforms demonstrated that membranous RANKL levels were significantly increased in the L-OA osteoblasts compared to normal and $\mathrm{H}-\mathrm{OA} .{ }^{71}$ The gene expression level of the RANKL1 profile followed the same trend as the membranous RANKL level. RANKL3 gene expression was lower on the H-OA osteoblasts compared to the normal and the L-OA osteoblasts; however, the overall outcome favoured RANKL1, hence the localization of RANKL at the membrane. Treatment of the L-OA osteoblasts with the osteotropic factors $1 \alpha, 25$ dihydroxyvitamin $\mathrm{D}_{3}$, IL- $1 \beta$, TNF- $\alpha$, PGE $_{2}$, and IL- 6 showed a significant increase in membranous RANKL and the RANKL1 gene expression level followed the same trend with the exception of treatment with TNF- $\alpha$. Upon treatment with TNF- $\alpha$, the increased membranous RANKL and the absence of effect on the RANKL1 gene expression strongly suggest the modulation of the other RANKL isoforms. Of great interest is that in the H-OA subpopulation, the above osteotropic factors had no effect on either membranous RANKL or its isoforms. Such findings demonstrate that each of the human OA subchondral bone subpopulations (L-OA and H-OA) has attained a different metabolic state; L-OA being enriched with factors promoting bone resorption and $\mathrm{H}-\mathrm{OA}$ having reduced resorptive proper- 


\section{Osteoarthritic Subchondral Bone Osteoblasts}

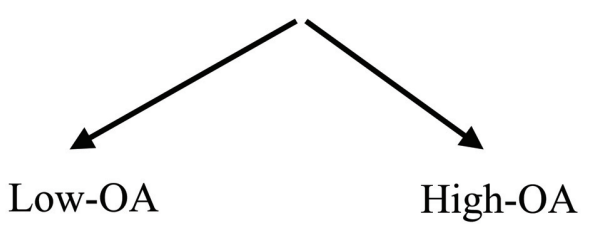

$\begin{array}{ccc}\begin{array}{c}\text { PGE } 2 \text { level } \\ \text { OPG/RANKL } \\ \text { ratio }\end{array} & \text { Low } & \text { High } \\ \begin{array}{ccc}\text { Osteoclastogenesis } \\ \begin{array}{c}\text { Subchondral } \\ \text { bone thickness }\end{array}\end{array} & \text { High } & \text { High } \\ & & \text { Low } \\ & & \text { Increase }\end{array}$

Fig. 4 Osteoarthritic (OA) subchondral bone osteoblasts can be discriminated into two subpopulations, Low-OA or High-OA, based on their endogenous production of $\mathrm{PGE}_{2}$. Differences between these two categories of osteoblasts include the OPG/RANKL ratio, the capacity to induce osteoclast formation, and the correlation to the thickness of OA subchondral bone.

ties, with the metabolism of the latter cells favouring bone formation. Thus, in humans, the presence of L-OA and $\mathrm{H}-\mathrm{OA}$ subchondral bone osteoblasts could reflect different stages of attempts to repair this damaged tissue in this disease: an increase in bone resorption followed by bone formation.

\section{OPG and RANKL as a Specific Therapeutic Target during Osteoarthritis}

$\mathrm{OA}$ is a disease that tends to get worse over time as the cartilage continues to wear away. Unfortunately, no curative therapeutics (that is, agents that stop the disease progression) are currently available for this disease. The main properties of the available treatment are to control the pain and improve the joint's ability to function. Some risk factors have been described for the development of $\mathrm{OA}$ and they include age, family history, excessive weight, joint injury, and as shown recently by magnetic resonance imaging, meniscal extrusion and bone marrow edema. ${ }^{85-89}$ Some non-medication therapies are also a very important component of the treatment of OA. These include moderate exercise to reduce pain and stiffness and to maintain or increase muscle tone, the application of heat and cold to provide relief from muscle and joint pain and a well-balanced diet, which is also essential for maintaining a healthy weight.

Medications commonly prescribed for OA consist of non-steroidal anti-inflammatory drugs (NSAIDs), as well as corticosteroids and viscosupplementation. Patients are also sometimes given complementary therapy including glucosamine and chondroitin sulfate. Finally, even if a patient is undergoing therapy, when OA becomes severe, surgery remains the last option. The most common surgical procedures for OA include hip and knee replacement.

Recently, the Glucosamine/chondroitin Arthritis Intervention Trial (GAIT) reported, following exploratory analyses, that the combination of glucosamine and chondroitin sulfate was effective on symptoms in a subpopulation of OA patients with moderate to severe knee pain. ${ }^{90}$ Interestingly, a recent in vitro study carried out on human L-OA subchondral bone osteoblasts demonstrated that the combination of glucosamine and chondroitin sulfate modulated the OPG/RANKL ratio, in which there was an increase in favour of reduced bone resorption. ${ }^{69}$ This was further supported by data showing that the combination of these compounds decreased the resorptive activity of L-OA osteoblasts. ${ }^{69}$ These data may help to explain how these two drugs exert a positive effect on OA pathophysiology.

\section{Conclusion}

In conclusion, this review emphasizes that the internalization and degradation of OPG is dependent on the presence of membranous RANKL or on cells expressing high levels of syndecan-1. The inhibitory effect of OPG on RANKL can be explained by its role not only as a decoy receptor but also as a modulator of RANKL halflife. Hence, as membranous RANKL controls the bioavailability of exogenous OPG, the equilibrium between RANKL and OPG is crucial for future therapeutic use of OPG. An ideal therapeutic approach would be to maintain an optimal soluble OPG concentration associated with a low internalization process.

Data also point to specific molecular mechanisms that 
appear to be operating during human OA progression. Studies demonstrate that the abnormal OPG and RANKL levels in human OA subchondral bone osteoblasts are dependent on the metabolic state of these cells and that the different abnormal expression of OPG and RANKL indicates different stages of attempts to repair the damaged subchondral bone.

Future therapeutic approaches could target reducing bone remodelling changes that take place in human OA subchondral bone, and data suggest that the modulation of OPG and RANKL could be an interesting target for such treatment. Since L-OA patients are in a remodelling/ resorptive phase, the inhibitory effect of OPG on bone resorption could therefore be proposed for this subpopulation; however, even though the administration of OPG in vivo in a murine OA model seems effective for the treatment of $\mathrm{OA},{ }^{73}$ care should be taken since full-length OPG is known to be a survival factor for tumor cells. ${ }^{91}$ Moreover, the anti-apoptotic effect of OPG via TRAIL can promote OA synovial membrane hyperplasia. An alternative approach is to inhibit RANKL by using an antiRANKL antibody or by acting on upstream factors responsible for the increased membranous localization of RANKL, for example by modulating one of the RANKL isoforms. In regard to the OA subpopulation, the addition of OPG or the inhibition of RANKL would be beneficial on the subchondral bone of the L-OA as this tissue is in a resorptive phase. However, in the H-OA patients, antiresorptive agents are expected to be less effective since the subchondral bone was shown to be in a formation phase. Nonetheless, further experiments exploring the effects of adding or increasing OPG or inhibiting RANKL on the subchondral bone pathophysiological pathways of each OA subpopulation are required.

\section{Acknowledgments}

The authors are grateful to Virginia Wallis from the Osteoarthritis Research Unit of the University of Montreal Hospital Centre for her assistance with the manuscript preparation.

\section{References}

1. Ng KW, Romas E, Donnan L, Findlay DM: Bone biology. Baillieres Clin Endocrinol Metab 1997; 11:1-22

2. Hill PA: Bone remodelling. Br J Orthod 1998;25:101-107

3. Troen BR: Molecular mechanisms underlying osteoclast formation and activation. Exp Gerontol 2003; 38:605-614

4. Franz-Odendaal TA, Hall BK, Witten PE: Buried alive: how osteoblasts become osteocytes. Dev Dyn 2006; 235:176-190

5. Kearns AE, Khosla S, Kostenuik PJ: Receptor activator of nuclear factor kappaB ligand and osteoprotegerin regulation of bone remodeling in health and disease. Endocr Rev 2008; 29:155-192

6. Khosla S: Minireview: the OPG/RANKL/RANK system. Endocrinology $2001 ; 142: 5050-5055$

7. Kwan Tat S, Padrines M, Theoleyre S, Heymann D, Fortun Y: IL-6, RANKL, TNF-alpha/IL-1: interrelations in bone resorption pathophysiology. Cytokine Growth Factor Rev 2004;15:49-60

8. Darnay BG, Haridas V, Ni J, Moore PA, Aggarwal BB: Characterization of the intracellular domain of receptor activator of NFkappaB (RANK). Interaction with tumor necrosis factor receptorassociated factors and activation of NF-kappaB and c-Jun N-terminal kinase. J Biol Chem 1998; 273:20551-20555

9. Armstrong AP, Tometsko ME, Glaccum M, Sutherland CL, Cosman D, Dougall WC: A RANK/TRAF6-dependent signal transduction pathway is essential for osteoclast cytoskeletal organization and resorptive function. J Biol Chem 2002; 277:4434744356

10. Gravallese EM, Goldring SR: Cellular mechanisms and the role of cytokines in bone erosions in rheumatoid arthritis. Arthritis Rheum 2000; 43:2143-2151

11. Cremer I, Dieu-Nosjean MC, Marechal S, Dezutter-Dambuyant C, Goddard S, Adams D, et al.: Long-lived immature dendritic cells mediated by TRANCE-RANK interaction. Blood 2002; 100:3646 $-3655$

12. Wiethe C, Dittmar K, Doan T, Lindenmaier W, Tindle R: Enhanced effector and memory CTL responses generated by incorporation of receptor activator of NF-kappa B (RANK)/RANK ligand costimulatory molecules into dendritic cell immunogens expressing a human tumor-specific antigen. J Immunol 2003; 171:4121-4130

13. Yu Q, Gu JX, Kovacs C, Freedman J, Thomas EK, Ostrowski MA: Cooperation of TNF family members CD40 ligand, receptor activator of NF-kappa B ligand, and TNF-alpha in the activation of dendritic cells and the expansion of viral specific CD8+ T cell memory responses in HIV-1-infected and HIV-1-uninfected individuals. J Immunol 2003; 170: 1797-1805

14. Bucay N, Sarosi I, Dunstan CR, Morony S, Tarpley J, Capparelli $\mathrm{C}$, et al:: Osteoprotegerin-deficient mice develop early onset osteoporosis and arterial calcification. Genes Dev 1998; 12: 1260 1268

15. Cardus A, Panizo S, Parisi E, Fernandez E, Valdivielso JM: Differential effects of vitamin D analogs on vascular calcification. J Bone Miner Res 2007; 22: 860-866

16. Orita Y, Yamamoto H, Kohno N, Sugihara M, Honda H, Kawamata $\mathrm{S}$, et al.: Role of osteoprotegerin in arterial calcification: development of new animal model. Arterioscler Thromb Vasc Biol 2007;27:2058-2064

17. Tan KB, Harrop J, Reddy M, Young P, Terrett J, Emery J, et al.: Characterization of a novel TNF-like ligand and recently described TNF ligand and TNF receptor superfamily genes and their constitutive and inducible expression in hematopoietic and nonhematopoietic cells. Gene 1997;204:35-46

18. Simonet WS, Lacey DL, Dunstan CR, Kelley M, Chang MS, Luthy $\mathrm{R}$, et al.: Osteoprotegerin: a novel secreted protein involved in the regulation of bone density. Cell 1997;89:309-319

19. Yasuda H, Shima N, Nakagawa N, Mochizuki SI, Yano K, Fujise $\mathrm{N}$, et al.: Identity of osteoclastogenesis inhibitory factor (OCIF) and osteoprotegerin (OPG): a mechanism by which OPG/OCIF inhibits osteoclastogenesis in vitro. Endocrinology 1998;139:1329 $-1337$

20. Gao YH, Shinki T, Yuasa T, Kataoka-Enomoto H, Komori T, Suda $\mathrm{T}$, et al.: Potential role of cbfa1, an essential transcriptional factor for osteoblast differentiation, in osteoclastogenesis: regulation of mRNA expression of osteoclast differentiation factor (ODF) . Biochem Biophys Res Commun 1998;252:697-702

21. Hofbauer LC, Dunstan CR, Spelsberg TC, Riggs BL, Khosla S: Osteoprotegerin production by human osteoblast lineage cells is stimulated by vitamin $\mathrm{D}$, bone morphogenetic protein-2, and cytokines. Biochem Biophys Res Commun 1998;250:776-781

22. Hofbauer LC, Gori F, Riggs BL, Lacey DL, Dunstan CR, Spelsberg TC, et al.: Stimulation of osteoprotegerin ligand and inhibition of osteoprotegerin production by glucocorticoids in human 
osteoblastic lineage cells: potential paracrine mechanisms of glucocorticoid-induced osteoporosis. Endocrinology 1999;140:4382 $-4389$

23. Lee SK, Lorenzo JA: Parathyroid hormone stimulates TRANCE and inhibits osteoprotegerin messenger ribonucleic acid expression in murine bone marrow cultures: correlation with osteoclastlike cell formation. Endocrinology 1999;140:3552-3561

24. Brandstrom H, Bjorkman T, Ljunggren O: Regulation of osteoprotegerin secretion from primary cultures of human bone marrow stromal cells. Biochem Biophys Res Commun 2001;280:831-835

25. Saika M, Inoue D, Kido S, Matsumoto T: 17beta-estradiol stimulates expression of osteoprotegerin by a mouse stromal cell line, ST-2, via estrogen receptor-alpha. Endocrinology 2001;142:2205 $-2212$

26. Thirunavukkarasu K, Miles RR, Halladay DL, Yang X, Galvin RJ, Chandrasekhar S, et al:: Stimulation of osteoprotegerin (OPG) gene expression by transforming growth factor-beta (TGF-beta). Mapping of the OPG promoter region that mediates TGF-beta effects. J Biol Chem 2001;276:36241-36250

27. Kong YY, Yoshida H, Sarosi I, Tan HL, Timms E, Capparelli C, et al.: OPGL is a key regulator of osteoclastogenesis, lymphocyte development and lymph-node organogenesis. Nature 1999;397:315-323

28. Hakeda Y, Kobayashi Y, Yamaguchi K, Yasuda H, Tsuda E, Higashio $\mathrm{K}$, et al.: Osteoclastogenesis inhibitory factor (OCIF) directly inhibits bone-resorbing activity of isolated mature osteoclasts. Biochem Biophys Res Commun 1998;251:796-801

29. Kanazawa K, Kudo A: Self-assembled RANK induces osteoclastogenesis ligand-independently. J Bone Miner Res 2005;20:20532060

30. Lee ZH, Kim HH: Signal transduction by receptor activator of nuclear factor kappa B in osteoclasts. Biochem Biophys Res Commun 2003;305:211-214

31. Kanazawa K, Azuma Y, Nakano H, Kudo A: TRAF5 functions in both RANKL- and TNFalpha-induced osteoclastogenesis. J Bone Miner Res 2003;18:443-450

32. Kanazawa K, Kudo A: TRAF2 is essential for TNF-alpha-induced osteoclastogenesis. J Bone Miner Res 2005;20:840-847

33. Iotsova V, Caamano J, Loy J, Yang Y, Lewin A, Bravo R: Osteopetrosis in mice lacking NF-kappaB1 and NF-kappaB2. Nat Med 1997;3:1285-1289

34. Li J, Sarosi I, Yan XQ, Morony S, Capparelli C, Tan HL, et al.: RANK is the intrinsic hematopoietic cell surface receptor that controls osteoclastogenesis and regulation of bone mass and calcium metabolism. Proc Natl Acad Sci U S A 2000;97:1566-1571

35. Matsuo K, Galson DL, Zhao C, Peng L, Laplace C, Wang KZ, et al.: Nuclear factor of activated T-cells (NFAT) rescues osteoclastogenesis in precursors lacking c-Fos. J Biol Chem 2004;279:26475-26480

36. Takayanagi H: The role of NFAT in osteoclast formation. Ann N Y Acad Sci 2007;1116:227-237

37. Lacey DL, Timms E, Tan HL, Kelley MJ, Dunstan CR, Burgess T, et al.: Osteoprotegerin ligand is a cytokine that regulates osteoclast differentiation and activation. Cell 1998; 93: 165-176

38. Yasuda H, Shima N, Nakagawa N, Yamaguchi K, Kinosaki M, Mochizuki S, et al.: Osteoclast differentiation factor is a ligand for osteoprotegerin/osteoclastogenesis-inhibitory factor and is identical to TRANCE/RANKL. Proc Natl Acad Sci U S A 1998; 95: 3597-3602

39. Collin-Osdoby P, Rothe L, Anderson F, Nelson M, Maloney W, Osdoby P: Receptor activator of NF-kappa B and osteoprotegerin expression by human microvascular endothelial cells, regulation by inflammatory cytokines, and role in human osteoclastogenesis. J Biol Chem 2001; 276: 20659-20672

40. Suzuki J, Ikeda T, Kuroyama H, Seki S, Kasai M, Utsuyama M, et al.: Regulation of osteoclastogenesis by three human RANKL isoforms expressed in NIH3T3 cells. Biochem Biophys Res Commun
2004; 314: $1021-1027$

41. Ikeda T, Kasai M, Utsuyama M, Hirokawa K: Determination of three isoforms of the receptor activator of nuclear factor-kappaB ligand and their differential expression in bone and thymus. Endocrinology 2001; 142: 1419-1426

42. Ikeda T, Kasai M, Suzuki J, Kuroyama H, Seki S, Utsuyama M, et al.: Multimerization of the receptor activator of nuclear factorkappaB ligand (RANKL) isoforms and regulation of osteoclastogenesis. J Biol Chem 2003; 278: 47217-47222

43. Lam J, Nelson CA, Ross FP, Teitelbaum SL, Fremont DH: Crystal structure of the TRANCE/RANKL cytokine reveals determinants of receptor-ligand specificity. J Clin Invest 2001; 108: 971-979

44. Ito S, Wakabayashi K, Ubukata O, Hayashi S, Okada F, Hata T: Crystal structure of the extracellular domain of mouse RANK ligand at 2.2-A resolution. J Biol Chem 2002; 277: 6631-6636

45. Lum L, Wong BR, Josien R, Becherer JD, Erdjument-Bromage H, Schlondorff J, et al.: Evidence for a role of a tumor necrosis factor-alpha (TNF-alpha)-converting enzyme-like protease in shedding of TRANCE, a TNF family member involved in osteoclastogenesis and dendritic cell survival. J Biol Chem 1999; 274: 13613 $-13618$

46. Schlondorff J, Lum L, Blobel CP: Biochemical and pharmacological criteria define two shedding activities for TRANCE/OPGL that are distinct from the tumor necrosis factor alpha convertase. J Biol Chem 2001; 276: 14665 -14674

47. Chesneau V, Becherer JD, Zheng Y, Erdjument-Bromage H, Tempst P, Blobel CP: Catalytic properties of ADAM19. J Biol Chem 2003; 278: 22331-22340

48. Hikita A, Kadono Y, Chikuda H, Fukuda A, Wakeyama H, Yasuda $\mathrm{H}$, et al.: Identification of an alternatively spliced variant of Ca2+promoted Ras inactivator as a possible regulator of RANKL shedding. J Biol Chem 2005; 280: 41700-41706

49. Lynch CC, Hikosaka A, Acuff HB, Martin MD, Kawai N, Singh RK, et al.: MMP-7 promotes prostate cancer-induced osteolysis via the solubilization of RANKL. Cancer Cell 2005; 7: 485-496

50. Lee Y, Schwarz E, Davies M, Jo M, Gates J, Wu J, et al.: Differences in the cytokine profiles associated with prostate cancer cell induced osteoblastic and osteolytic lesions in bone. J Orthop Res 2003; 21: $62-72$

51. Standal T, Seidel C, Hjertner O, Plesner T, Sanderson RD, Waage $\mathrm{A}$, et al:: Osteoprotegerin is bound, internalized, and degraded by multiple myeloma cells. Blood 2002; 100: 3002-3007

52. Mosheimer BA, Kaneider NC, Feistritzer C, Djanani AM, Sturn $\mathrm{DH}$, Patsch JR, et al.: Syndecan-1 is involved in osteoprotegerininduced chemotaxis in human peripheral blood monocytes. J Clin Endocrinol Metab 2005; 90: 2964-2971

53. Tat SK, Padrines M, Theoleyre S, Couillaud-Battaglia S, Heymann D, Redini F, et al:: OPG/membranous-RANKL complex is internalized via the clathrin pathway before a lysosomal and a proteasomal degradation. Bone 2006; 39: 706-715

54. Theoleyre S, Kwan Tat S, Vusio P, Blanchard F, Gallagher J, Ricard-Blum S, et al.: Characterization of osteoprotegerin binding to glycosaminoglycans by surface plasmon resonance: role in the interactions with receptor activator of nuclear factor kappaB ligand (RANKL) and RANK. Biochem Biophys Res Commun 2006; 347: 460-467

55. Akatsu T, Murakami T, Ono K, Nishikawa M, Tsuda E, Mochizuki SI, et al.: Osteoclastogenesis inhibitory factor exhibits hypocalcemic effects in normal mice and in hypercalcemic nude mice carrying tumors associated with humoral hypercalcemia of malignancy. Bone 1998; 23: 495-498

56. Min H, Morony S, Sarosi I, Dunstan CR, Capparelli C, Scully S, et al.: Osteoprotegerin reverses osteoporosis by inhibiting endosteal osteoclasts and prevents vascular calcification by blocking a process resembling osteoclastogenesis. J Exp Med 2000; 192: 463 $-474$

57. Nakashima T, Wada T, Penninger JM: RANKL and RANK as 
novel therapeutic targets for arthritis. Curr Opin Rheumatol 2003; 15: $280-287$

58. Vanderkerken K, De Leenheer E, Shipman C, Asosingh K, Willems A, Van Camp B, et al.: Recombinant osteoprotegerin decreases tumor burden and increases survival in a murine model of multiple myeloma. Cancer Res 2003; 63: 287-289

59. Bekker PJ, Holloway DL, Rasmussen AS, Murphy R, Martin SW, Leese PT, et al.: A single-dose placebo-controlled study of AMG 162 , a fully human monoclonal antibody to RANKL, in postmenopausal women. J Bone Miner Res 2004; 19: 1059-1066

60. Martel-Pelletier J, Lajeunesse D, Pelletier JP: Etiopathogenesis of osteoarthritis. In: Koopman WJ, Moreland LW, eds. Arthritis \& Allied Conditions. A Textbook of Rheumatology. 15th ed. Baltimore: Lippincott, Williams \& Wilkins, 2005, pp. 2199-2226

61. Lajeunesse D, Massicotte F, Pelletier JP, Martel-Pelletier J: Subchondral bone sclerosis in osteoarthritis: Not just an innocent bystander. Modern Rheumatology 2003; 13: 7-14

62. Pelletier JP, Boileau C, Brunet J, Boily M, Lajeunesse D, Reboul $\mathrm{P}$, et al.: The inhibition of subchondral bone resorption in the early phase of experimental dog osteoarthritis by licofelone is associated with a reduction in the synthesis of MMP-13 and cathepsin K. Bone 2004; 34: 527-538

63. Agnello KA, Trumble TN, Chambers JN, Seewald W, Budsberg SC: Effects of zoledronate on markers of bone metabolism and subchondral bone mineral density in dogs with experimentally induced cruciate-deficient osteoarthritis. Am J Vet Res 2005; 66: $1487-1495$

64. Brandt KD, Myers SL, Burr D, Albrecht M: Osteoarthritic changes in canine articular cartilage, subchondral bone, and synovium fifty-four months after transection of the anterior cruciate ligament. Arthritis Rheum 1991; 34: 1560-1570

65. Hilal G, Martel-Pelletier J, Pelletier JP, Ranger P, Lajeunesse D: Osteoblast-like cells from human subchondral osteoarthritic bone demonstrate an altered phenotype in vitro: Possible role in subchondral bone sclerosis. Arthritis Rheum 1998; 41: 891-899

66. Hilal G, Martel-Pelletier J, Pelletier JP, Duval N, Lajeunesse D: Abnormal regulation of urokinase plasminogen activator by insulin-like growth factor 1 in human osteoarthritic subchondral osteoblasts. Arthritis Rheum 1999; 42: 2112-2122

67. Massicotte F, Lajeunesse D, Benderdour M, Pelletier J-P, Hilal G, Duval N, et al.: Can altered production of interleukin $1 \beta$, interleukin-6, transforming growth factor- $\beta$ and prostaglandin E2 by isolated human subchondral osteoblasts identify two subgroups of osteoarthritic patients. Osteoarthritis Cartilage 2002; 10: 491-500

68. Massicotte F, Fernandes JC, Martel-Pelletier J, Pelletier JP, Lajeunesse D: Modulation of insulin-like growth factor 1 levels in human osteoarthritic subchondral bone osteoblasts. Bone 2006; 38: 333-341

69. Kwan Tat S, Pelletier JP, Vergés J, Montell E, Lajeunesse D, Fah$\mathrm{mi} \mathrm{H}$, et al.: Chondroitin and glucosamine sulfate in combination decrease the pro-resorptive properties of human osteoarthritis subchondral bone osteoblasts. Arthritis Res Ther 2007; 9: R117

70. Kwan Tat S, Pelletier JP, Lajeunesse D, Fahmi H, Lavigne M, Martel-Pelletier J: The differential expression of osteoprotegerin $(\mathrm{OPG})$ and receptor activator of nuclear factor kappaB ligand (RANKL) in human osteoarthritic subchondral bone osteoblasts is an indicator of the metabolic state of these disease cells. Clin Exp Rheumatol 2008; 26: 295-304

71. Tat SK, Pelletier JP, Lajeunesse D, Fahmi H, Duval N, MartelPelletier J: Differential modulation of RANKL isoforms by human osteoarthritic subchondral bone osteoblasts: influence of osteotropic factors. Bone 2008; 43:284-291

72. Komuro H, Olee T, Kuhn K, Quach J, Brinson DC, Shikhman A, et al.: The osteoprotegerin/receptor activator of nuclear factor kappaB/receptor activator of nuclear factor kappaB ligand system in cartilage. Arthritis Rheum 2001; 44: 2768-2776

73. Shimizu S, Asou Y, Itoh S, Chung UI, Kawaguchi H, Shinomiya
$\mathrm{K}$, et al.: Prevention of cartilage destruction with intraarticular osteoclastogenesis inhibitory factor/osteoprotegerin in a murine model of osteoarthritis. Arthritis Rheum 2007; 56: 3358-3365

74. Takayanagi H, Iizuka H, Juji T, Nakagawa T, Yamamoto A, Miyazaki $\mathrm{T}$, et al.: Involvement of receptor activator of nuclear factor kappaB ligand/osteoclast differentiation factor in osteoclastogenesis from synoviocytes in rheumatoid arthritis. Arthritis Rheum 2000; 43: 259-269

75. Saidenberg-Kermanac'h N, Cohen-Solal M, Bessis N, De Vernejoul MC, Boissier MC: Role for osteoprotegerin in rheumatoid inflammation. Joint Bone Spine 2004; 71: 9-13

76. Kmiec Z, Sokolowska I: [Role of tumor necrosis factor family ligands in the pathogenesis of rheumatoid arthritis--new therapeutical opportunities]. Pol Merkur Lekarski 2007; 22: 300-304

77. Gravallese EM, Manning C, Tsay A, Naito A, Pan C, Amento E, et al.: Synovial tissue in rheumatoid arthritis is a source of osteoclast differentiation factor. Arthritis Rheum 2000; 43: 250-258

78. Adamopoulos IE, Danks L, Itonaga I, Locklin RM, Sabokbar A, Ferguson DJ, et al.: Stimulation of osteoclast formation by inflammatory synovial fluid. Virchows Arch 2006; 449: 69-77

79. Redlich K, Hayer S, Maier A, Dunstan CR, Tohidast-Akrad M, Lang S, et al.: Tumor necrosis factor alpha-mediated joint destruction is inhibited by targeting osteoclasts with osteoprotegerin. Arthritis Rheum 2002; 46: 785-792

80. Romas E, Sims NA, Hards DK, Lindsay M, Quinn JW, Ryan PF, et al.: Osteoprotegerin reduces osteoclast numbers and prevents bone erosion in collagen-induced arthritis. Am J Pathol 2002; 161: $1419-1427$

81. Ritchlin CT, Haas-Smith SA, Li P, Hicks DG, Schwarz EM: Mechanisms of TNF-alpha- and RANKL-mediated osteoclastogenesis and bone resorption in psoriatic arthritis. J Clin Invest 2003;111:821-831

82. Ritchlin CT, Schwarz EM, O'Keefe RJ, Looney RJ: RANK, RANKL and OPG in inflammatory arthritis and periprosthetic osteolysis. J Musculoskelet Neuronal Interact 2004; 4: 276-284

83. Takemura M, Harada A, Mizuno M, Yano K, Yamada Y: Relationship between osteoprotegerin/osteoclastogenesis inhibitory factor concentration in synovial fluid and disease severity in individuals with osteoarthritis of the knee. Metabolism 2001; 50: 1-2

84. Skoumal M, Kolarz G, Haberhauer G, Woloszczuk W, Hawa G, Klingler A: Osteoprotegerin and the receptor activator of NF-kappa B ligand in the serum and synovial fluid. A comparison of patients with longstanding rheumatoid arthritis and osteoarthritis. Rheumatol Int 2005; 26: $63-69$

85. Berthiaume MJ, Raynauld JP, Martel-Pelletier J, Labonté F, Beaudoin G, Bloch DA, et al.: Meniscal tear and extrusion are strongly associated with the progression of knee osteoarthritis as assessed by quantitative magnetic resonance imaging. Ann Rheum Dis 2005; 64: 556-563

86. Raynauld JP, Martel-Pelletier J, Berthiaume MJ, Beaudoin G, Choquette D, Haraoui B, et al.: Long term evaluation of disease progression through the quantitative magnetic resonance imaging of symptomatic knee osteoarthritis patients: correlation with clinical symptoms and radiographic changes. Arthritis Res Ther 2006; 8: R21

87. Ding C, Martel-Pelletier J, Pelletier JP, Abram F, Raynauld JP, Cicuttini $\mathrm{F}$, et al:: Meniscal tear as an oseoarthritis risk factor in a largely non-osteoarthritic cohort: A cross-sectional study. J Rheumatol 2007; 34: 776-784

88. Pelletier JP, Raynauld JP, Berthiaume MJ, Abram F, Choquette D, Haraoui B, et al.: Risk factors associated with the loss of cartilage volume on weight bearing areas in knee osteoarthritis patients assessed by qualitative MRI: A longitudinal study. Arthritis Res Ther 2007; 9: R74

89. Raynauld J-P, Martel-Pelletier J, Berthiaume M-J, Abram F, Choquette $\mathrm{D}$, Haraoui $\mathrm{B}$, et al.: Correlation between bone lesion changes and cartilage volume loss in patients with osteoarthritis of 
the knee as assessed by quantitative magnetic resonance imaging over a 24-month period. Ann Rheum Dis 2008; 67: 683-638

90. Clegg DO, Reda DJ, Harris CL, Klein MA, O'Dell JR, Hooper $\mathrm{MM}$, et al.: Glucosamine, chondroitin sulfate, and the two in combination for painful knee osteoarthritis. N Engl J Med 2006; 354: $795-808$
91. Fisher JL, Thomas-Mudge RJ, Elliott J, Hards DK, Sims NA, Slavin $\mathrm{J}$, et al.: Osteoprotegerin overexpression by breast cancer cells enhances orthotopic and osseous tumor growth and contrasts with that delivered therapeutically. Cancer Res 2006; 66: $3620-$ 3628 